\title{
Review on Pulpy Kidney Disease
}

\author{
Dinsefa Jemal ${ }^{*}$, Mohazeba Shifa and Bedaso Kebede
}

Veterinary Drug and Animal Feed Administration and Control Authority, Ministry of Livestock and Fisheries, Addis Ababa, Ethiopia

\begin{abstract}
Pulpy kidney disease is a poisoning produce by a toxin from gram positive spore forming an obligate anaerobic rod bacterium called Clostridium perfringens type $\mathrm{D}$. The disease is perhaps the best known pathogenic Clostridium perfringens type, widely regarded as the causative organism of fatal enterotoxaemia of sheep or "over eating disease". It appears to have a worldwide distribution and produce epsilon toxin that damages endothelial cells, which is almost exclusively responsible for the host pathology and subsequent death. The toxin is produced in the gut by abundantly growing bacterial cells and is triggered by some feeding factors and grazing management when animals are switched from a poor to a rich pasture or when a days with temperate to warm weather. This causes annual grasses to growth rapidly, with low fibre and high ammonia contents. This toxin absorbed to the systemic circulation. The disease is clinically manifested by acute, sub-acute and chronic neurologic condition, characterized by sudden death or neurologic and respiratory signs including blindness, convulsions, bleating, frothing by the mouth and recumbence before death. It is diagnosed by the detection of specific anti-bodies and necropsy finding. Treatment, prevention and control for the disease depends on anti-toxin, supportive treatment and correction of dietary excessively and vaccination. Therefore animals should be protected from over feeding. In addition an epidemiological surveillance on the disease should be done in animals and dietary management, so that appropriate prevention and control strategies can be implemented.
\end{abstract}

Keywords: Clostridium perfringens type D; Pulpy kidney disease; Sheep

Abbreviations: $\mu \mathrm{m}$ : Micrometer; \%: Percent; ELISA: EnzymeLinked Immuno Sorbent Assay; LD: Lethal Dose; ML: Milliliter; MNT: Mouse Neutralization Test; PCR: Polymerase Chain Reaction; W/V: Weight by Volume

\section{Introduction}

Pulpy kidney disease is a disease caused by Clostridium perferingens type D, occurs in sheep worldwide. The condition is also described as "over eating disease "because gorging on a high grain diet or on succulent pasture predisposes to its development. In ingestion of excessive quantities of food leads to "carry over" of partially digested food from the rumen in to the intestine [1]. This disease is perhaps the best known pathogenic Clostridium perfringens type, being widely regarded as the causative organism fatal enterotoxaemia of sheep or "over eating disease". It appears to have a worldwide distribution but is not common intestinal commensal. It produces epsilon toxin that damages endothelial cells, which is almost exclusively responsible for the host pathology and subsequent death. The toxin is produced in the gut by abundantly growing bacterial cells and is triggered by some feeding factors and absorbed in the systemic circulation. The epsilon toxin is resistant to digestive enzymes; in fact, these enzymes convert the freshly secreted less active a prototoxin into the fully toxic form. Clinically, when large amounts of epsilon toxin are produced in the gut, its absorption in to the systemic circulation increases capillary permeability in many organs and tissues, including intestinal mucosa. This increases it absorption rate and consequently the systemic effects leading to excessive renal damage, hyperglycemia, hypertension and edema in various organs, including the brain [2].

Lesions of Clostridium perfringens type D infections consist of multi systemic hemorrhages, particularly of serosal surfaces; pericardial effusion is present along with mild gastroenteritis [3]. Clostridial toxins have been identified by serum neutralization on laboratory animals (Mice and Guinea pigs) using specific anti-sera. This toxin typing requires a continuous supply of laboratory animals and the employment of monovalent anti-body for diagnostic sera, and it is difficult to find and extremely expensive. Compared with this classical technique, the polymerase chain reaction method has been shown to be much more rapid, with results obtained in a few hours, and it is much more reliable. The prophylaxis of enterotoxaemia in animals is achieved with vaccination; the polymerase chain reaction technique can thus become a first choice tool for identification and typing of the Clostridium perfringens strains which cause these diseases. In turn, this simplifies the development of vaccines adapted to the epidemiological situation [4].

Vaccination history is frequently used by animal owners and veterinarians to rule out infections by Clostridium perfringens. However, the quality of Clostridium perfringens vaccines varies greatly between countries and manufacturers, and vaccines are not always correctly transported, stored and or administered. In addition, individual variation in antibody responses between animals occurs frequently in both sheep and goats [5]. Sheep's are protected against the disease when vaccines of high immunologic power and adequate immunization strategies are use, and remain protected for a year when a booster dose is applied. 28-42 days after the first vaccination produces lower and shorter lived titers than the sheep and the animas require booster dose every 3-4 months throughout their live after the first double vaccinations [6]. The objective of this review is to review pulpy kidney disease.

*Corresponding author: Dinsefa Jemal, Veterinary Toxicology Analyst, Veterinary Drug and Animal Feed Administration and Control Authority, Ministry of Livestock and Fisheries, Addis Ababa, Ethiopia, Tel: +251937875237/+251114717258; Fax: +114717239; E-mail: mohazeba@gmail.com

Received May 27, 2016; Accepted July 13, 2016; Published July 18, 2016

Citation: Jemal D, Shifa M, Kebede B (2016) Review on Pulpy Kidney Disease. J Vet Sci Technol 7: 361. doi:10.4172/2157-7579.1000361

Copyright: ( 2016 Jemal D, et al. This is an open-access article distributed under the terms of the Creative Commons Attribution License, which permits unrestricted use, distribution, and reproduction in any medium, provided the original author and source are credited. 


\section{Pathogenic Significance}

Clostridium perfringens type $\mathrm{D}$ is the etiology that causes the pulpy kidney diseases in the small ruminants. This disease prevents the absorption of the nutrients in the intestine, rumen and abomasum's by producing the epsilon toxin which damages the endothelial cells of these tissues. This toxin cause capillary damage even in the kidney, lungs and brain resulting in edema.

\section{Etiology}

The causative agent is Clostridium perfringens type D. However, predisposing factors are also essential; the most common of these is the ingestion of excessive amounts of feed or milk in the very young and of green in feed lot lambs. In young lambs, the diseases usually is restricted to sing lambs, because of a ewe with twins seldom gives enough milk to allow enterotoxaemia to develop. In the feed lot, the disease usually is seen in lambs switched rapidly to high-grain diets. As starch intake increases, it provide suitable medium for growth of causative bacteria which produces epsilon toxin. A major effect of the toxin is to cause vascular damage, particularly of capillaries in the brain. Sheep carry strains of Clostridium perfringens type $\mathrm{D}$ as the part of normal micro flora of intestine and serves as the source of organisms to infect the new born most such carrier, have non vaccinal antitoxin titers [1].

Enterotoxaemia caused by epsilon toxin-producing Clostridium perfringens type $\mathrm{D}$ is an important syndrome in sheep and goat industries. Grain fed lambs on a high concentrate diet is most susceptible, but adult sheep and goat also may be affected [7]. The epsilon toxin increase vascular permeability, leading to edema in the pulmonary system and kidneys and explaining the name pulpy kidney disease [8].

Enterotoxaemia caused by Clostridium perfringens type D, a disease of major importance in sheep and of lesser important in cattle and goats, is caused by strain of the bacterium that produce the epsilon toxin. This is type not common soil organism as is type A but may be isolated from the faces of apparently normal sheep and, less frequently, cattle [9]. The toxin is secreted as a pro to toxin, which is activated by proteolytic cleavage. The cleaved part reassociates with the active toxin molecule to give the active toxin a conformational and serologic similarity to the proto toxin [10].

The most important among all Clostridium perfringens caused diseases that occur in sheep, cattle and goats. The lambs and calves are more affected. The toxins cause diffused reddening of mucosa and sub endocardial as well as myocardial hemorrhage. Animal may be come blind due to glycosuria. Straw colored fluid is found in the peritoneal, pleura and pericardial sacs. Toxin also affects the central nervous system bringing about behavioral changes [11].

\section{Classification of Toxin}

Enterotoxaemia is caused by Clostridium perfringens that are gram-positive rod. It produces at least twelve different kinds of toxins and antigens, many of which are important in the production of the various diseases. The species is divided in to type A to $\mathrm{E}$ on the basis of antigenic differences and lethal toxins. Some of them are as follows: Alpha: This is the principal toxin produced in varying amounts by all Clostridium perferingens. It is also histolytic and necrotizing. Beta: This toxin is produced mostly by type $\mathrm{B}$ and $\mathrm{C}$ perfringens. It is responsible for causing enteritis in cattle, sheep and humans. Epsilon: This toxin is produced by type B and D and is only slightly toxic. This toxin is necrotizing and highly lethal. Theta: It is a lethal, hemolytic, necrotizing toxin produced by strains of types A, B, C, D and E. Iota-This toxin is produced only by type $\mathrm{E}$ strains. Kappa-This toxin produced by all types of Clostridium perfringens and is responsible for the softening and pulping of affected tissues. Lambda: This toxin is produced by strains of type $\mathrm{B}$ and $\mathrm{E}$. $\mathrm{Mu}$ : It is hydrolyzing hyaluronic acid is produced by all types, except type B [11].

\section{Morphology and Staining Reaction}

Clostridium perfringens occurs as thick, straight-sided rods, either singly or in pairs, seldom in chains. The individual cells are about one micrometer wide and four to eight micro meters long. The spores are oval and small enough not to cause much swelling of the rods. Spores do not form in highly acid media; hence they are unlikely to be found in media that contain fermentable carbohydrate. Strains vary in their ability to sporulate; sometimes it is difficult to find spores no matter what the nature of culture media. Sporulation in tissue is uncommon. In old cultures Clostridium perfringens is pleomorphic, appearing as clubbed types, ballooned cells, and filaments. Capsules are formed in tissues and in some types of culture media. There are no flagella. The organisms of young cultures retain gram's stain; Older organisms frequently decolorized [12].

\section{Cultural and Biochemical features}

Colonies of Clostridium perfringens on blood agar are surrounded by an inner zone of complete hemolysis and an outer zone of incomplete hemolysis. On egg-yolk agar, an opaque, whitish precipitate is formed with in the medium as a result of the activity of lecithinase (phospholipids c). In deeper agar the colonies are small and biconvex. In a tube containing glucose or other fermentable substrates, the medium is fragmented and even blown out of the tube by the abundant amounts of gases that are formed. Clostridium perfringens rapidly liquefies gelatin but not coagulated egg medium or Loffler's blood serum [12].

\section{Immunological Aspects}

Immunity is antibody mediated correlates with antitoxin levels. Immunizing preparations often include bacterial components as well. Passive and active immunization is important in the control of the disease antitoxin given to sick animals and those at risk, protection the lasts two up to three weeks prophylactic dosages, given subcutaneously, can be doubled and given intravenously for therapy. Active immunization of dams with two injections of bacterin-toxoid combinations prior to parturition ensures nurslings passive protection for the first weeks of life. During out breaks, antitoxin and toxoid are often given and a second dose of toxoid is administered some weeks later. Protection of lambs against type $\mathrm{D}$ enterotoxaemia requires two vaccinations at monthly intervals. The course should be completed two weeks before the lambs are placed on full feed [13].

\section{Epidemiology}

\section{Occurrence}

The disease is occurs worldwide. Clostridium perfringens type $\mathrm{D}$ is an obligate parasite of the intestinal tract but under certain conditions it proliferates and produces large quantities of toxins which can be lethal. Heavy grain diet or lush pastures have been found to be favorable for the proliferation of the bacteria and occurrence of the disease. Thus, the disease commonly affects well fed animals especially in intensive feed lot units. Factors which result in intestinal stasis or slow the passage of the ingesta through the intestines such as heavy tape worm 
infestations favor the accumulation of the toxin and occurrence of the disease. Inclement weather, coccidiossis and de worming can predispose animals to the disease. Lambs and kids of three to twelve weeks and six to twelve months old have been found to be the most susceptible groups.

Enterotoxaemia associates with Clostridium perfringens type $\mathrm{D}$ is a disease of ruminant animals, primarily of lambs and is worldwide in distribution. The common practiced of vaccination against this disease is has reduced in its prevalence but it is still a common disease. While most common in lambs, it is also an important disease of calves and goats. It occurs rarely in adult cattle, deer domesticated camels, and possibly horses. In pastured sheep, it causes heavy losses, particularly in flocks managed for the production of lamb and mutton. The prevalence in flocks varies a great deal but seldom exceeds ten percent. The case fatality rate approximates hundred percent. In North America enterotoxaemia ranks as own of the main causes of loss among feedlot lambs [7].

In a survey in two feel lots the disease had an annual prevalence of $3.14 \%$ and $1.49 \%$; it ranked third in importance as a cause of death despite a policy of vaccination, the casts of prevention programs were the largest expenditure of all disease prevention program in the feed lots.

\section{Source of Infection and Mode of Transmission}

Strains of the bacteria are commonly found in soil and manures under normal circumstances, where animals ingest the bacteria, this is not a disease issue as it is often simply excreted. However, when lambs ingest a high quality of milk, feed, pasture, or all the three, the bacteria population can increase in numbers rapidly. Thus the common name "over eating" disease. Often, the type D strain more commonly affects single lambs rather than twins. These lambs receive more milk and are often stronger and more aggressive eaters. What actually kills the lambs is an endotoxin secreted by the bacteria. Enterotoxaemia is not a contagious disease in that the presence of the causative bacteria in the intestine does not in itself produce the disease. Under natural conditions the ingestion of feed contaminated by infected faces introduces the organism into the alimentary tract but the disease does not occur unless other factors intercede [14].

\section{Risk Factors}

\section{Age affected}

Pulpy kidney is a disease which can affect sheep at any age, but is most frequent in lambs of 4-10 weeks of age and in fattening stock from which 6 months to 1 years of age. It is caused by the rapid multiplication of Clostridium perfringens type $\mathrm{D}$ in the small intestines and the subsequent absorption of the epsilon toxin, which is produced by the organisms in the form of anon-toxic protoxin and converted to a lethal toxin by the action of trypsin [15].

Pulpy kidney disease is per acute and of a short duration, with most cases being found dead. Those found a live show neurological signs and diarrhea is seen in longer-living cases. Affected animals don not recover. Sheep's changed from a low to a high plane of nutrition may be vulnerable to the pulpy kidney disease, as the rumen microbial flora have not adjusted sufficiently rapidly and partly digested food containing carbohydrate passes in the small intestine. This allows Clostridium perfringens to multiply rapidly, producing large amounts of epsilon protoxin, which is converted to lethal toxin by the action of trypsin [16]. Thriving lambs 3-10 weeks of age are commonly affected. The course of the disease is usually short and lambs are often found dead [1].

\section{Environmental and husbandry risk factor}

Clostridium perfringens typed normally inhabits the alimentary tract of sheep and other ruminants but only in small numbers. The extent to which it occurs in the alimentary tract varies widely in between flocks, although this accounts' only in part for the variable prevalence. The organism does not persist for more than 1 year in the soil. Under certain conditions; the organisms proliferate rapidly in the intestines and produce lethal quantities of epsilon toxin. In most, it not all circumstances, the affected animals are on highly nutritious diets and are in very good condition. The husbandry conditions in which the disease occurs include grazing on lush, rapidly growing pasture or young cereal crops, and heaving grain feeding in fed lots. Lambs on well-fed, heavy milking ewes are particularly susceptible. The occurrence of the disease under these conditions has given rise to the name "over eating disease" [1].

\section{Pathogenesis and Clinical Finding}

\section{Pathogenesis}

Clostridium perfringens type D organisms are abundant in the ileum and less so in other parts of small intestine. Sudden change from low to high energy and especially starchy diets favors rapid multiplication of the saccharolytic Clostridium perfringens type D which produces an epsilon toxin. The toxin is endotheliotropic and binds to the endothelial cells causing damage. Damage to the capillary endothelium results in increased permeability of the intestinal mucosa and this facilitate further absorption of the epsilon and other toxins. The toxin also causes capillary damage in other tissues such as kidneys, lungs and the brain resulting in edema. Extracellular edema in the brain in associated with nervous signs.

In the normal course of events, ingested Clostridium perfringens type $\mathrm{D}$ are destroyed in large numbers in the rumen and abomasum's, although some survive to reach the duodenum, where multiplication occurs and toxin is produced. Toxemia does not occur because the movement of ingesta keeps the bacteria population and toxin content dawn to a low level. In certain circumstances, this does not hold and multiplication of the organisms and the production of toxin proceed to the point where toxemia occurs. One of the circumstance has been shown to be the passage of large quantities of starch granules in to the duodenum when sheep over eat on grain diets or are changed suddenly from a ration consisting largely of roughage to one consisting mainly of grain. Other factors such as heavy milk feeding may have the same effect. A slowing of alimentary tract movement has also been thought to permit excess toxin accumulation and it may be that any factor that causes intestinal stasis will pre disposes to the disease [7].

In most case the ingested Clostridium perfringens type D, attaches itself to the surface of the epithelial cell of the intestinal villus and produces toxins which are responsible for disease in previously a tonic young ones. Initially there is damage to the microvillus with the destruction and desquamation of the intestinal epithelial cells and the production of hemorrhagic enteritis. The absorbed toxin circulates to various vital organs to produce cellular damage, hemorrhage and necrosis [11].

\section{Clinical findings}

Sudden death the principal manifestation of per acute disease in young animals although some of the animals may be dull, depressed and anorexia. In acute cases, there is frothy salivation, green or pasty diarrhea, staggering, recumbence, opisthotonus, colonic convulsions coma and death. Colic and bloat may occur. Affected adult sheep often 
lag behind the rest of the flock and show nervous signs as in young animals which include hypersensitivity, staggering gait, ataxia and knuckling of the fetlock. Champing of the jaws, blindness, salivation, rapid and shallow respiration, atonic rumen and pasty faces may also be evident. In goats, the acute disease is characterized by diarrhea or dysentery, abdominal discomfort and convulsions. Death may occur within 24-36 hours of onset of the disease. In both sheep and goats, the sub-acute syndrome is characterized by anorexia, intermittent diarrhea or dysentery or the presence of epithelial shreds in faces. The chronic disease is characterized by progressive weight loss, emaciation anemia but goats may also be diarrheic. The chronic nervous form of the disease is characterized by aimless wandering, in coordination, paralysis of the master muscles in appetence and ruminal atony.

In lambs, the course of the illness is very short, often less than 2 hours and never more than 12 hours, and many are found dead without previously manifesting signs. In closed observed flocks the first signs may be dullness, depression, yawning, facial movements and loss of interest in feed. Acute case may show little more than severe colonic convulsions with frothing at the mouth and sudden death. Cases which survive for a few hours show a green, pasty diarrhea and staggering. The temperature is usually normal but may be elevated if the convulsions are severe. In calves and adult cattle the syndrome is similar to that seen in adult sheep, with nervous signs predominating. Per acute cases are found dead without having shown premonitory signs of illness and with no evidence of struggling. The more common, acute cases show a sudden onset of bellowing, mania and convulsions, the convulsions persisting until death occurs 1-2 hours. Sub-acute cases, many of which recover, do not drink, are quiet and docile and appear to be blind although the eye preservation reflex persists [14].

\section{Diagnosis}

The diagnosis of a disease is based on the epidemiological features especially the type of diet, clinical and pathological features. Gram positive Clostridium perfringens type rods can be demonstrated from smears of the ingesta or intestinal lesions isolation of the bacteria by culturing a sample of fecal material and demonstration of epsilon toxin in intestinal contents can be highly suggestive of the disease. Protection of mice injected with toxin filtrates from the ingesta using specific anti-serum for the epsilon is also used to confirm the disease. Other diagnostic tests include ELISA, counter immunofluorescence, passive haemagglutination and immuno diffusion.

The mere presence of Clostridium perfringens type D or of its epsilon toxin in the intestinal contents is insufficient evidence for a diagnosis type $\mathrm{D}$ enterotoxaemia. This is so because of the wide spread occurrence of type D strains and because epsilon toxin may normally be present in the intestine but is not absorbed by the naturally resistant or immune animal. Diagnosis of type D enterotoxaemia must be based on: large numbers of Clostridium perfringens type D in the intestine, epsilon toxin in the small intestine, type D organisms in the kidney and other parenchymatous organs at death, and Sugar in the urine [17].

Diagnosis depends up on history of ruminal atony due to predisposing. Define d clinical manifestations of respective disease also help in diagnosis. Necropsy findings, animal inoculation test and identification of bacteria confirm the case [11].

\section{Necropsy Finding}

At necropsy, the carcass is in good condition and no gross changes are observed in per acute disease. The acute syndrome is characterized by presence of a clear straw colored fluid in the pericardial sac which rapidly clots on exposure to air, patchy congestion of the abomasal and intestinal mucosa and, presence of custard ingesta in the intestines. Diffusion petechial hemorrhages occur on the peritoneal surface of the abomasum's and intestines. Petechial or ecchymotic hemorrhages also occur in the muscles of the flank, muscular portion of the diaphragm, epicardium and in the thymus. There is rapid decomposition of the carcass and purple discoloration of the hairless areas of the body. The small intestine is distended with gas. Adak congested liver with hemorrhagic spots on its surface and gelatinous or blood tinged pericardial fluid are observed for few hours after death. The kidney has a mottled appearance, soft consistency and the cortex is jelly-like or semi fluid (pulpy like). Nephrosis, congestion of the renal cortex and rapture of capillaries occur. In young animal's terminal rapture of the abomasum's has been reported. In goats, the acute disease is characterized by pulmonary edema, necrosis of the small and large intestinal walls. The intestinal contents may be green, blood stained or mucoid and, fibrinous casts or stands may present the lumen of large intestine. The mesenteric lymph nodes are edematous. In histological sections of the brain, the presence of per vascular edema, hemorrhages and bilateral symmetrical areas of leucoencephalomalacia in the basal ganglia, thalamus, substantial nigra and cerebellar peduncles is characteristics of the nervous form of the disease (also known as focal symmetrical encephalomalacia).

Post mortem lesions are in consistent. The "pulpy kidney" lesion may not be seen in freshly examined specimens and is not considered d to be a use full diagnostic finding. The pericardium, serous, thymus, and diaphragm may have small areas of hemorrhage. The pericardial sac often contains excess fluid. The lungs may be edematous. Glucosuria is considered a hall mark of the type D enterotoxaemia in cattle and sheep. In goats, fibrino hemorrhagic enterocolitis is the most consistent finding [18]. The condition of animal is usually good but there is often fecal staining of then perineum and the rapid decomposition the carcass. In per acute cases there may be no gross lesions. More frequently, there is an excess of clear, straw-colored pericardial and thoracic fluid that clots on exposure to air. Many petechiae are present in the epicardium and endocardium and there is pulmonary edema. Patchy congestion of the abomasal and intestinal mucosae is usual and the small intestine often contains a moderate amount of thin, creamy ingesta. The content of large intestine may be watery and dark green [7]. The characteristic finding of soft pulpy kidneys is only use full in animals necropsied within a few hours after death, as it is nonspecific and merely correlates to a more rapid rate of autolysis. Microscopy of experimentally induced ovine type D enterotoxaemia cases confirms that the renal change represents autolysis and not a true nephrosis [19]. Hemorrhagic lesions are seen on necropsy in the peritoneum, pericardium, endocardium, diaphragm, abomasum's and duodenum. There is softening of kidney also [11].

\section{Laboratory Diagnosis}

The toxins of Clostridium perfringens are detected and identified by serum neutralizing tests in mice and guinea pigs. The procedures are described by Carter [20]. Samples of the intestine content (small intestine) and other body fluids should be collected as soon as after death possible and $1 \%$ chloroform added as a preservative. Samples should be kept chilled during transport to the laboratory, where they are centrifuged. The supernatant $(0.2-0.4 \mathrm{ml})$, then injected intravenously into mice and intradermally into white skin areas of guinea pigs. Parts of the sample should be treated with trypsin ( $1 \%$ trypsin powder/v) and left at room temperature for 1 hour to activate epsilon and iota toxins and destroy $\beta$-toxins. The enzyme treated solutions are them injected 
into second group of mice and pigs. If the toxins present, they die in 4-12 hours [12].

\section{Toxin detection}

The most widely accepted criterion on which to base definitive diagnosis of type $\mathrm{D}$ enterotoxaemia in both sheep and goats is detection of epsilon toxin in intestinal contents [21]. Low concentrations of epsilon toxin (250 lethal dose in 50/ml) maybe found in the small intestine of clinically healthy sheep [22]. Although in other studies, no epsilon toxin was found in intestinal content of healthy sheep's [19]. There are no reports of presence or absence of epsilon toxin in the intestine of normal goats. If diagnosis only based on toxicologic results, it is potentially possible to misdiagnose type $B$ (produces $\alpha$ toxin and $\varepsilon$ toxin) infections as type $\mathrm{D}$ (produce $\alpha$ toxin and $\varepsilon$ toxin $s$ if $\beta$ toxin is destroyed by the action of intestinal trypsin. However, type B infections are rare, and gross and histologic lesions of type $\mathrm{D}$ disease can be very characteristic of this form of enterotoxaemia. Several techniques are available for detecting epsilon toxin in intestinal contents or other body fluids and in culture supernatants. Among these are the MNT, ELISA and counter immune electrophoresis [22].

\section{Ancillary}

Ancillary tests for diagnosis of type $\mathrm{D}$ enterotoxaemia in sheep and goats have been described [23]. The most useful is detection of urinary glucose, the presence of which in any amount is strongly suggestive of enterotoxaemia in both sheep and goats. However, this finding is not consistent [24] and although glycosuria is a useful positive predictor of type D enterotoxaemia, absence of glycosuria does not rule out a diagnosis of enterotoxaemia in either species. Care should be taken to consider the clinical history, in particularly any previous parenteral administration of glucose, which produces high levels of glycosuria [20].

\section{Differential Diagnosis}

The differential diagnosis of pulpy kidney disease in young animals includes acute ruminal impaction, poliencephalomalacia, other clostridia enterotoxaemia and acute pasteurellosis. No convolutions are observed in acute ruminal impaction and the course of the disease is longer (1-3 days) and polioencephalomalacia takes a longer course. Acute pasteurelosis can be differentiated by isolation and identification of pasteurella species from the affected tissues. In adult animal's rabies, acute lead poisoning and pregnancy toxemia should be considered in the differential diagnosis of focal symmetrical encephalomalacia. In rabies there will be a history of encounter with the rabid animal or bite wounds. The presence of materials containing lead such as paints can be suggestive of lead poising. Pregnancy toxemia occurs during the late pregnancy in under in adequate nutrition and ketouria is common feature at clinical pathology.

\section{Treatment}

Treatment of the animals is usually in effective, due to the rapid progression of the disease in the animal. The key to treatment therefore, is preventing the disease. Antitoxins produced by commercial companies can be given either orally or by injection. Administration of antibiotics such as penicillin may be recommended, but it frequently of a little value after clinical signs appear. Hyper immune serum is an efficient short term prophylactic but is unlikely to be of much value in sick animal's because of the acute nature the disease, although serum (50 $\mathrm{ml}$ twice daily) combined with orally administered sulphadimidine is reported to be effective in goats [25].

\section{Methods of Control and Prevention}

The most effective way of controlling clostridial diseases is by vaccination. The veterinary surgeon or the manufacturer's data sheets should be consulted for their proper use. Organic standards permit the use of vaccination in cases where there is known disease risk. Single or four in one vaccines are preferred to more complex multiple vaccines unless such cover is specifically required. Vaccine choice and use should be agreed with the nominated veterinary surgeon to ensure adequate disease protection during the conversion period with, where possible, progressive reductions in use as the organic unit become established. Only healthy animals should be vaccinated [26]. The lower level of animal production under organic flock management will help to prevent the enterotoxaemia to a large extent. This is specifically the case for pulpy kidney, which is due to over feeding with carbohydrates. A combined antitoxin preparation against lamb dysentery, pulpy kidney and struck is available (Lambisan, Hoechst Roussel Vet) for passive immunization of at-risk sheep and lambs. The preparation may be expected to confer passive immunity for 3-4 weeks $[27,28]$. Vaccination should be combined with careful management of the flock. In the case of enterotoxaemia this should include gradual introduction of sheep to a higher level of nutrition in order to allow rumen flora to adjust. When cases of enterotoxaemia occur in sheep on a high plane of nutrition, the diet should be changed to feed containing lower levels of carbohydrate [16].

\section{Conclusion and Recommendations}

The causative agent for pulpy kidney disease is a toxin produced by Clostridium perfringens type $\mathrm{D}$. The toxin could be produced in the gastro intestinal content. The spores of Clostridium perfringens type D germinate under anaerobic condition. There is no geographic limitation for the occurrence of the disease. But the source of exposure to the toxin and risk to the disease differ between seasons because of the difference in feed availability in the year and feeding management practice. During the treatment of this disease, anti-toxins were considered to be essential for increasing the success rate. Since Clostridium perfringens type $\mathrm{D}$ is ubiquitous in nature, eradication of the disease was reported to be impossible but necessitates control by vaccination. On view of the above conclusion the following recommendation should be intended:

- Decrease in food volume, and increase in the frequency of feeding.

- Increase in animal movement.

- Vaccination should be administered at the proper age and time recommended during which the risk of toxicities are high.

- The predisposing factor should be well identified and increase fiber in the feed.

- Avoiding heavy tape worm infestation

- Avoiding of de worming

\section{Acknowledgements}

Authors would like to express our sincere gratitude to all whom help us in technical and ideological without felling any tiredness for the accomplishment of this review.

\section{Funding}

No funding was received for this review.

\section{References}

1. Quinn PJ, Carter GR, Markey BK, Donnelly WJ, Leonard F (2002) Veterinary Microbiology and Microbial disease. 2nd edn, Blackwell Publishing Company, USA 66: 92-93. 
2. Niilo L (1980) Clostridium perfringens in Animal Disease: A Review of Current Knowledge. Can Vet J 21: 141-148.

3. McGavin MD, Zachary JF (2007) Pathologic Basis of Veterinary disease. Elsevier Science Health Science Division. Saint Louis: Mosby Inc., USA, p: 1488.

4. Kadra B, Guillou JP, Popoff M, Bourlioux P (1999) Typing of sheep clinical isolates and identification of enterotoxigenic Clostridium perfringens strains by classical methods and by polymerase chain reaction (PCR). FEMS Immunol Med Microbiol 24: 259-266.

5. Blackwell TE, Butler DG (1992) Clinical signs, treatment, and postmortem lesions in dairy goats with enterotoxemia: 13 cases (1979-1982). J Am Vet Med Assoc 200: 214-217.

6. Uzal FA, Kelly WR (1999) Serum antibody responses to a Clostridium perfringens epsilon toxoid vaccine in goats. Anaerobe 5: 287-289.

7. Radostitis OM, Gay CC, Hinchcliff KW, Constable PD (2007) Veterinary Medicine: A Textbook of the Diseases of Cattle, Sheep, Pigs, Goats, and Horses. 10th edn. Elsevier Saunders, USA, p: 842.

8. Michelsen PGE (1996) Disease caused by toxins of Clostridium perfringens. In: Large Animal Internal Medicine. Smith BP (ed) Elsevier - Health Sciences Division, St. Louis Mosby Inc., USA, p: 262.

9. Itodo AE, Adesiyun AA, Adekeye JO, Umoh JU (1986) Toxin-types of Clostridium perfringens strains isolated from sheep, cattle and paddock soils in Nigeria. Vet Microbiol 12: 93-96.

10. McDone J (1986) Toxins of Clostridium perfringens type A-E. In: Pharmacology of Bacterial Toxins. Dorner F, Drews J (eds.). Elsevier Science \& Technology Books, Oxford, England 119: 477-517.

11. Upadhayay AK (2005) Text of Preventive Veterinary Medicine. 1st edn. IBDC Publishing, India, pp: 59-61.

12. Timoney JF, Gillespie JH, Scott FW, Barlough JE (1988) Hagnon and Bruner's Microbiology and Infectious Diseases of Domestic Animal. 8th edn. A division of Cornell University Press, Ithaca and London, pp: 223-229.

13. Hirsh DC, MacLachlan NJ, Walker RL (2004) Veterinary Microbiology. 2nd edn Black Well Publishing, p: 202.

14. Blood DC, Henderson JA (1974) Veterinary Medicine. 4th edn. Baillieretindal London, UK, p: 132.
15. Buven JJ (1970) Role of toxin in the host parasite relationships. In: Microbial Toxin. Aji SJ, Kadis S, Montie TC (eds.). Academic Press, New York, USA, pp 223-270.

16. Buxton D, Donachie W (1991) Clostrial diseases. In: Diseases of sheep. 2nd edn. Martin WB, Aitken and ID. Black Well Scientific Publishers, Oxford, pp: 104-114.

17. Sterne M, Thomson A (1991) The isolation and identification of clostridia from pathological conditions of animals. Bull Off Int Epizoot 59: 1487-1498.

18. Blackwell TE, Butler DG, Bell JA (1992) Enterotoxemia in the goat: the humoral response and local tissue reaction following vaccination with two different bacterin-toxoids. Can J Comp Med 47: 127-132.

19. Uzal FA, Kelly WR, Morris WE, Bermudez J, Baisón M (2004) The pathology of peracute experimental Clostridium perfringens type $D$ enterotoxemia in sheep. $\mathrm{J}$ Vet Diagn Invest 16: 403-411

20. Carter GR (1984) Diagnostic procedures in veterinary microbiology. Spring Field, pp: 191-192.

21. Uzal FA, Bodero DAV, Kelly WR (1998) Variability of serum antibody responses of goat kids to a commercial Clostridium perfringens epsilon toxoid vaccine. Veterinary Record 143: 472-474.

22. Bullen JJ, Batty II (1990) Enterotoxemia of sheep. Vet Rec 69: 1268-1276

23. Uzal FA, Kelly WR (1998) Experimental Clostridium perfringens type D enterotoxemia in goats. Vet Pathol 35: 132-140.

24. Uzal FA, Kelly WR, Thomas R, Hornitzky M, Galea F (2003) Comparison of four techniques for the detection of Clostridium perfringens type $D$ epsilon toxin in intestinal contents and other body fluids of sheep and goats. J Vet Diagn Invest 15: 94-99.

25. Shank PL (1990) Enterotoxemia of Sheep. Vet Rec 61: 262.

26. Batungbacal MR, Scott GR (1982) Suppression of the immune response to clostridial vaccine by tick-borne fever. J Comp Pathol 92: 409-413.

27. Bullen JJ (1992) Enterotoxaemia of sheep: Clostridium welchii type D in the alimentary tract of normal animals. J Pathol Bacteriol 62: 201-206.

28. Bishop YM (1998) The Veterinary Formulary. 4th edn. Pharmaceutical Press London, UK. 